\title{
Amyloids, Congo red and the apple-green effect
}

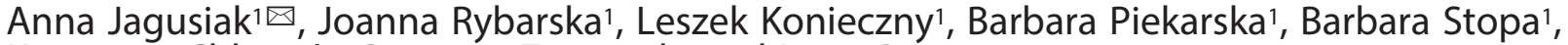 Katarzyna Chłopaś2, Grzegorz Zemanek¹ and Irena Roterman³}

${ }^{1}$ Chair of Medical Biochemistry, Jagiellonian University - Medical College, Kraków, Poland; 2Faculty of Medicine, Jagiellonian University - Medical College, Kraków, Poland; '3Department of Bioinformatics and Telemedicine, Jagiellonian University - Medical College, Kraków, Poland

\begin{abstract}
This paper attempts to find evidence of the previously proposed opinion that amyloids complex with Congo red molecules which preserve their supramolecular organization. As evidence of the overpowering tendency of Congo red molecules to self-assemble, we present an increasing acidity of molecules that follows increasing concentration of the dye, and a highly notable nonlinear increase in absorbance in the UV band (300-400 nm). This effect is analyzed in a model where the amyloid fibril is simulated by polyvinyl alcohol, providing a scaffold to stabilize a long Congo red micelle. Enormous absorbance in the UV band, coupled with the increasing association capabilities of individual Congo red molecules may cause the absorbance to extend even into the visible band. In addition, the UV and visual absorbance bands shift significantly, depending on conditions, and may either approach or recede from each other, leading to spectral changes which may be observed under polarized light. This commonly observed spectral variability appears to be associated with the strong capacity for electron delocalization in supramolecular Congo red complexed with amyloids.
\end{abstract}

Key words: amyloids, self-assembled molecules, Congo red, applegreen effect

Received: 16 September, 2018; revised: 25 February, 2019; accepted: 27 February, 2019; available on-line: 14 March, 2019

$\square_{\text {e-mail: anna.jagusiak@uj.edu.pl }}$

Acknowledgements of Financial Support: This work was financially supported by the National Science Centre, Poland (grant \# 2016/21/D/NZ1/02763)

Abbreviations: $\mathrm{BB}$, bromophenol blue; $\mathrm{CR}$, Congo red; $\mathrm{DLS}$, dynamic light scattering; EB, Evans blue; TY, Titan yellow; TB, Trypan blue; UV, ultra violet

\section{INTRODUCTION}

The relation between amyloids and notable pathologies has long been known, and amyloids themselves have been an object of studies for nearly 100 years. However, despite many in-depth analyses, their nature has not yet been revealed sufficiently.

Clearly, the ultimate object of research is to propose therapies which would prevent or counteract the formation of amyloids in living tissues. This, however, calls for a thorough understanding of the phenomenon.

In amyloid research, Congo red (CR) is very frequently used as a specific marker of amyloid fibrils (although the dye is also capable of binding to some other proteins, e.g. albumin and also to cellulose). Congo red self-associates in aqueous solvents, forming ribbon-like micellar structures. Analysis under polarized light of post-absorption emission spectra of complexes formed by Congo red and amyloids reveals optical activity which manifests itself as a green glow. This is referred to as the apple-green effect. Many authors consider it as evidence that Congo red has become complexed with fibrils, and in some cases the effect is even taken as a necessary condition for detection of the amyloid itself. An open issue is whether Congo red retains its supramolecular character when interacting with fibrils, or whether it binds as a collection of individual molecules, attached to specific groups in the amyloid (as it is often suggested) (Frid et al., 2007; Carter et al., 1998; Lendel et al., 2010; Wu et al., 2012; Ma et al., 2009; Klunk et al., 1989; Turnell et al., 1992). The presented paper attempts to resolve this issue.

A detailed explanation of the complexation mechanisms would also help us to better understand the nature of amyloids.

\section{MATERIALS AND METHODS}

Reagents. Congo red ( $97 \%$ purity) and Evans blue (EB), Titan yellow (TY) and Bromophenol blue (BB) were purchased from the Sigma Aldrich Chemical Company. Polyvinyl alcohol was purchased from Chempur Poland. Dextran was purchased from Sigma Aldrich. Nanotubes were purchased from the Sigma Aldrich Chemical Company. All reagents, including the abovementioned ones, were of analytical grade.

Laboratory equipment. Standard electrophoresis was used for analysis of the micellar nature of Congo red and other dyes, and to study the complexation capabilities of various matrices, such as polyvinyl alcohol.

Dynamic light scattering (DLS) method (DynaPro MS800 Protein Solutions Inc.) was applied in the measurements of hydrodynamic radius. Microscopic analysis was conducted using a Carl Zeiss Jena fluorescence microscope equipped with a polarizer extension.

The spectrophotometer used in the analysis was purchased from Hitachi Japan.

Experimental methods. Electrophoretic analyses on agarose and polyacrylamide gels were conducted under standard conditions. TrisHCl buffer (0.05 M, pH 8.0) was applied. Micellar nature of Congo red was revealed in electrophoresis on a polyacrylamide gel at $15 \%$ density. Dimension of structures formed by Congo red and other dyes was assessed by measuring dispersion (hydrodynamic radius) in DLS. However, molecular masses were not computed due to the unusual shape of these micellar structures.

Comparative dialysis was also conducted to measure the permeation capabilities of Congo red and bromophenol blue (BB) in a dialysis bag entirely saturated with 
Congo red. Both, the Congo red and bromophenol blue were used at concentrations of $10^{-6} \mathrm{M}$. The dyes were dissolved in a TrisHCl buffer $(0.05 \mathrm{M}, \mathrm{pH} 8.0,0.9 \%$ $\mathrm{NaCl}$ ) and dialyzed against $1000 \mathrm{ml}$ of the same buffer.

The spectra of Congo red and other dyes were analyzed in the UV and visual range. Because of variable positions of Congo red absorption bands on the wavelength scale, our discussion refers only to the wavelength range of 300-400 for UV and 400-600 for the visible light. The desired absorbances of Congo red in spectrophotometric analyses were obtained by adding specific quantities of a dye (in the microliter range) at initial concentrations of $2.5 \mathrm{mg} / \mathrm{ml}$ to $1 \mathrm{ml}$ of a suitable buffer. In case of $\mathrm{EB}$ and TB, a stock solution was used $(3.2 \mathrm{mg} / \mathrm{ml}$ concentration).

Polyvinyl alcohol was adjusted to $1 \%$ concentration in solutions used.

Amyloids were obtained by digestion of IgG light chains (Bence-Jones) with pepsin, in accordance with (Linke et al., 1973).

The spectrum of Congo red in complex with carbon nanotubes was obtained following sonication of nanotubes with Congo red and preparation of a solute from which the free (uncomplexed) fraction of Congo red was subsequently filtered out using PTFE membranes (Merck Millipore Ltd).

Spectra of control samples were obtained in $0.05 \mathrm{M}$ Tris/ $\mathrm{HCl} \mathrm{pH} 7.4,0.9 \% \mathrm{NaCl}$ buffer, and $0.05 \mathrm{M}$ acetate buffer ( $\mathrm{pH}$ 4.3).

Modeling complexation of Congo red by amyloids to acknowledge the apple-green effect. Mixtures formed by adding Congo red to polyvinyl alcohol or Dextran were dried on microscope slides under a warm airstream. This was followed by mechanically coercing the scaffold fibers in complex with Congo red to form a coherent structure by applying pressure that slowly shifted across the slide by using blunt metal or glass tools. The resulting optical activity was analyzed under polarized light.

\section{RESULTS AND DISCUSSION}

\section{Amyloid - ordered protein aggregation}

A well-documented stage of the formation of amyloid deposits is the transformation of native proteins into partially unfolded structures (Colon et al., 1992; Khurana et al., 2001). This is usually accompanied by exposure of nonpolar fragments of the polypeptide chain which under normal circumstances are packed in the central part of the protein (Kauzmann, 1959; Kalinowska et al., 2017; Dygut et al., 2016). This, in turn, leads to aggregation in an aqueous solvent. Spontaneous aggregation of denatured proteins, induced e.g. by increased temperature, typically results in amorphous aggregates (Chiti et al., 2017); however, in the case of amyloidogenic proteins the process is not chaotic and instead produces fibrillary structures. This fact alone appears inexplicable. A question emerges: what mechanism directs the aggregation of protein molecules to form coherent fibrils, despite the fact that amyloids involve proteins of varying origin?

The most popular explanation refers to the multistage process of amyloidogenesis, where formation of fibrils is preceded by formation of dimers or oligomers, limiting the structural possibilities for further aggregation while promoting linear assembly which eventually produce a fibril (Fig. 1) (Krishnan et al., 2012; Kayed et al., 2003; Modler et al., 2003; Harper et al., 1997; Nelson et al.,

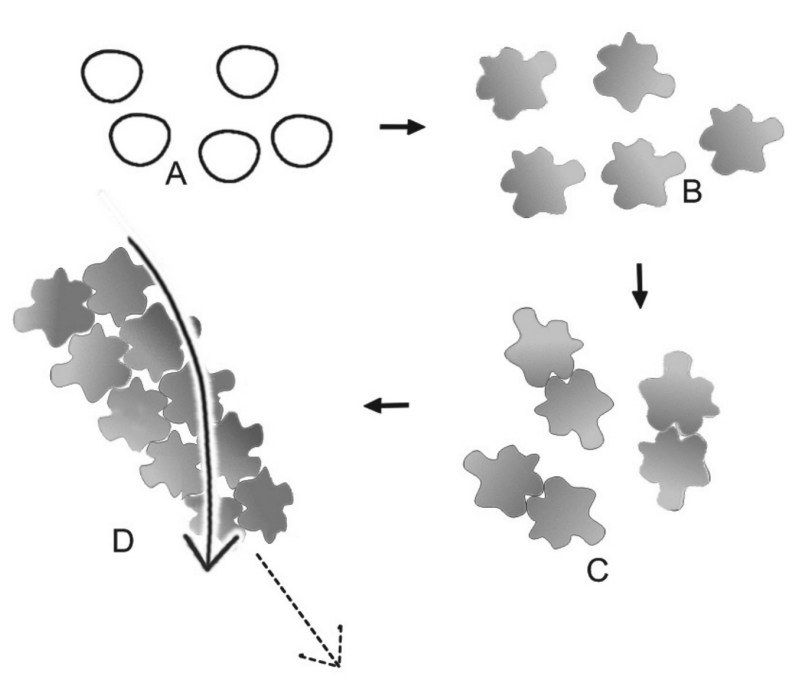

Figure 1. Model presenting the possible stepwise mechanism of amyloid fibril formation

A - native proteins, B - partly unfolded proteins, C - dimers or oligomers, D - fibrillation

2006; Pratim Bose et al., 2010; Serpell, 2000; Huang et al., 2006; Schormann et al., 1998).

While amyloidogenesis may also result in amorphous aggregations, fibrils are generally regarded as "typical" amyloid forms (Kayed et al., 2003). Notably, the fibril is also a highly durable structure.

In contrast to globular proteins, whose structure is an expression of the biological program encoded in their amino acids sequence, amyloids emerge as a result of uncontrolled, spontaneous aggregation.

\section{Congo red}

Congo red is a popular dye, but also a known amyloid stain. Chemically, it is a symmetrical, planar, elongated molecule with a nonpolar central part and distally placed polar groups. Dyes from this family which also include Evans blue and Trypan blue, among others (Fig. 2), exhibit a strong tendency to self-assemble, even though

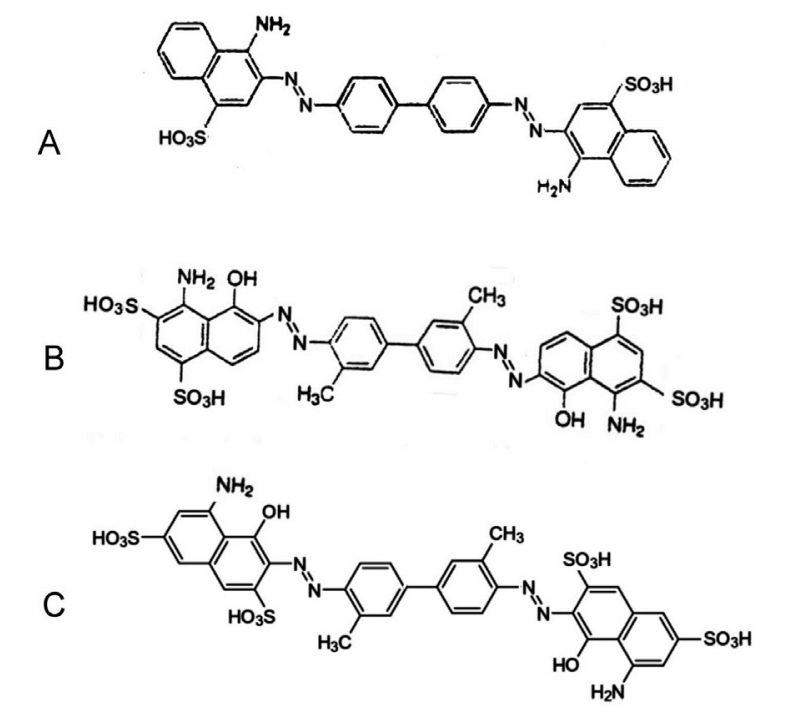

Figure 2. Chemical formulas of dyes with symmetrical, elongated molecules exhibiting different tendency to self-associate and form ribbon-like supramolecular structures.

A - Congo red (CR), B - Evans blue (EB), C - Trypan blue (TB) 


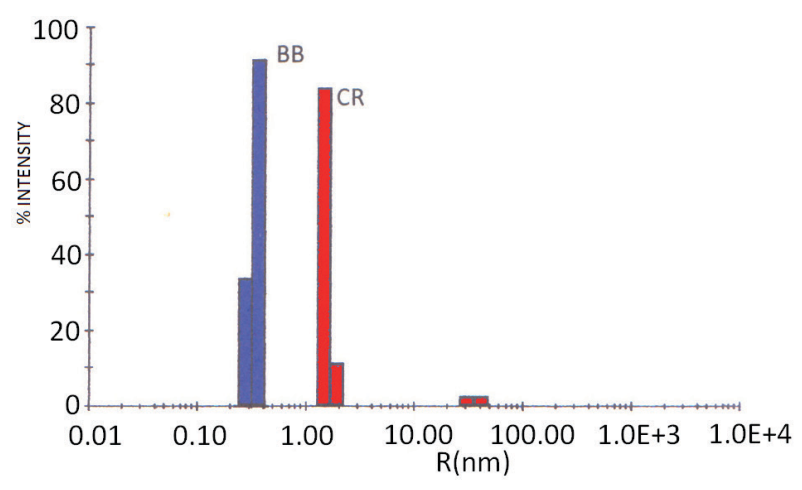

Figure 3. DLS analysis of Congo red (CR) and bromophenol blue (BB) - dyes with similar molecular mass and charge.

The indicated higher Congo red hydrodynamic radius $(R)$ reveals its micellar nature.

the cohesiveness of the resulting micelle is usually lower than in the case of Congo red. This may be caused by the presence of substituents which hamper close association, e.g. in Trypan blue. Self-associating dyes do not produce spherical micelles. Instead, they tend to adopt ribbon-like forms which is a consequence of their symmetrical structure (Skowronek et al., 1998; Herbst et al., 2018).

Given the ribbon-like character of the micelle, coupled with exposure of nonpolar surface, we may expect a dye in the self-assembled form to bond to different fibrillary structures. A basic question arises: do such dyes interact with amyloids or other proteins as coherent micelles or as individual molecules (Stopa et al., 2006; Roterman \& Konieczny, 2018).

The above question is of critical importance - if the specificity of complexation arises as a consequence of micellar properties, it would radically alter our views regarding the interaction of Congo red with amyloids. It would also pave the way towards use of this dye as a drug carrier, capable of targeted delivery of therapeutic compounds to areas where amyloid aggregates are found. This is enabled by noncovalent stabilization of supramolecular structures which provide a way to incorporate various external compounds via intercalation - a promising approach from the perspective of pharmacology (Jagusiak et al., 2018; Nummelin et al., 2017; Chen et al., 2015).

There is a growing evidence that Congo red interacts with amyloids in the described fashion. The specificity of this interaction can be explained by the physical properties of the dye micelle, rather than pairwise interactions between dye molecules and functional groups exposed by the amyloid.

Unlike in the "single molecule" interaction scenario, the mutual affinity of elongated fibrils and ribbon-like micelles is facilitated by large contact areas, supporting a multitude of weak (even nonspecific) interactions which combine to form a strong complex. Under these conditions specificity is underpinned by structural properties.

Congo red tends to form supramolecular structures in aqueous solvents. Thus, supramolecular interactions may be reasonably expected in the case of amyloids. Supramolecular Congo red in solution may be revealed e.g. by DLS measurements comparing the dispersion effects, which provides an indirect measure of the molecular masses of Congo red and bromophenol blue - dyes which share similar molecular masses and charge distributions, but differ with respect to self-association capa-

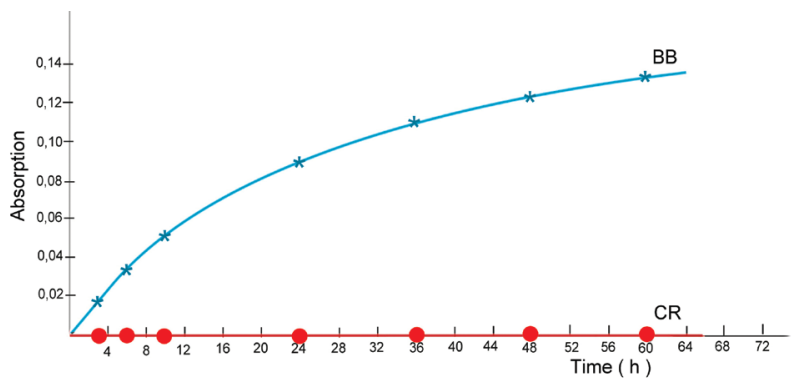

Figure 4. Evidence of the supramolecular nature of Congo red (CR) in dialysis of a mixture of bromophenol blue (BB) and Congo red.

Dialysis was performed in a cellulose bag saturated with Congo red. Of two used dyes, Congo red resists dialysis which confirms its micellar structure. The figure presents registration of dyes outside the bag by spectral absorption.

bilities. Unlike Congo red, BB is not a planar molecule, exhibiting very low propensity for self-association, particularly in an alkaline environment. In effect, it is usually found as a collection of individual molecules (Fig. 3).

For the reasons stated above, BB may be used as a control structure.

Supramolecular aggregations may be also detected by dialysis, which retains CR while allows $\mathrm{BB}$ to filter through (Fig. 4).

The presence of a stable supramolecular form of CR in aqueous solutions is also revealed by electrophoresis on dense $(15 \%)$ polyacrylamide gel. This process reveals significant differences in migration for both dyes, unlike agarose gels where both dyes migrate at very similar rates. It shows that CR, whose migration in polyacrylamide gels is decreased, is composed of larger entities, most likely due to this dye's micellar characteristics (Fig. 5).

It also turns out that strong association of Congo red molecules alters their physicochemical properties. Thus, self-association is not a purely mechanical phenomenon. Congo red electrophoresis on agarose gels indicates variability in migration depending on the dye concentration: higher concentration results in a stronger association and in consequence a faster migration. This evidences a change in the $\mathrm{pK}$ of polar groups, driven by progressive association of the dye molecules (Fig. 6) (Stopa et al., 2010).

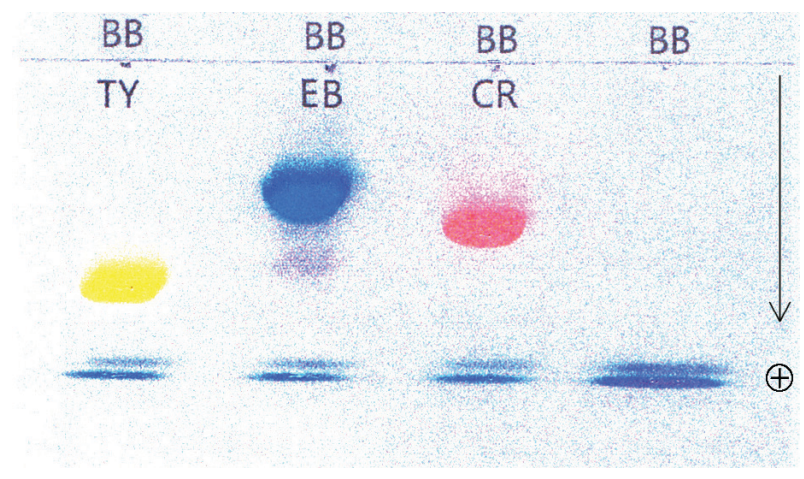

Figure 5. Reduced migration of Congo red (CR) when compared to bromophenol blue (BB) in polyacrylamide gel electrophoresis in a high density gel (15\%), showing that in contrast to $B B$, Congo red (as well as other self-assembled dyes, i.e. EB and TY), retains its micellar structure.

TY - Titan yellow. 


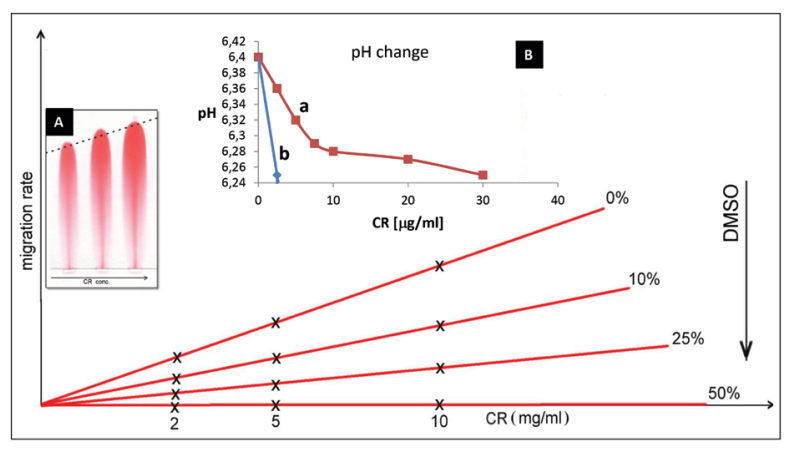

Figure 6 . The increase in Congo red migration rate along with the increasing concentration of the dye seen in electrophoretic analysis.

Straight lines in the main figure present this effect and its possible dampening by DMSO (used in the range of 0-50\%). Direct experimental presentation of this effect (fragment) is shown in Inset $A$, while Inset $B$ explains the reason for migrational dependence of the dye on concentration, as caused by variable acidity of molecules - $a . b-$ quick alteration of $\mathrm{pH}$ in the presence of polyvinyl alcohol (1\%) favouring self-assembly of the dye.

The above phenomenon, though unusual, points to a new and interesting property of Congo red: higher concentrations promote association, thereby increasing acidity of the dye. Association of Congo red proceeds by way of face-on contact, mainly between the aromatic rings located in the central, nonpolar part of the molecule. The process involves $\pi$-electrons which may travel along the ribbon-like micelle (if its length is sufficient) - a phenomenon referred to as the ring current. As a result, charges tend to congregate in the polar regions of the ribbon (Spólnik et al., 2011; Liu et al., 2016; Sahakyan et al., 2013), while the large central fragment is relatively devoid of electrons. This, in turn, triggers polarization of bonds and results in increased acidity. Elongation of the ribbon exacerbates this effect. Migration of electrons along the ribbon is perpendicular to the planar arrangement of individual molecules. On the other hand, individual Congo red molecules enable horizontal intra-molecular electron delocalization due to their resonance character and planar elongated structure. Only when molecules associate and form long ribbon-like supramolecular structures their electrons (mostly contributed by the central benzene rings) undergo inter-molecular delocalization which is perpendicular to the molecular plane. Therefore, a competition emerges between horizontal delocalization (determined by the dye's covalent structure) and delocalization which is perpendicular to the plane of the molecule (dependent on the non-covalent organization). Equilibrium may hence be affected by different external stimuli.

\section{Self-assembling visualization by spectral effects}

Inevitably, association affects the dye's spectrum, mostly in the UV range. The absorption band is present at $300-400 \mathrm{~nm}$. As it turns out, absorption in this band nonlinearly increases as the concentration of Congo red and its ordered structure increases, engaging more molecules in the absorption of light. It confirms the emergence of a new characteristic property of the dye and clearly reflects the effects of stronger association (Fig. 7 and Fig. 8). Variability involves not just the degree of absorbance, but also the location of maxima in the UV and visible bands (Fig. 9). These maxima were either closer or farther apart depending on analytic conditions. In our observations, the spacing of UV and visible absorption bands of Congo red oscillates in the range of

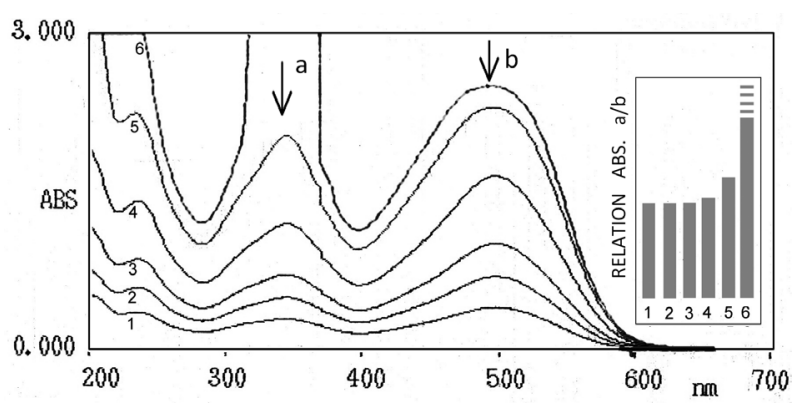

Figure 7. Rapid, nonlinear increase in absorbance (ABS) in the UV band (300-400 $\mathrm{nm}$ ) which occurs when the concentration of the dye increases.

Non-linear spectral changes occur as the ribbon-like micellar structure of Congo red grows longer. Inset - bars show increments of $a: b$ (relation of absorption) presented in conventional scale following the increasing concentration of a dye done by step-wise addition (1:6) of stock solution of the dye $(2.5 \mathrm{mg} / \mathrm{ml})$.
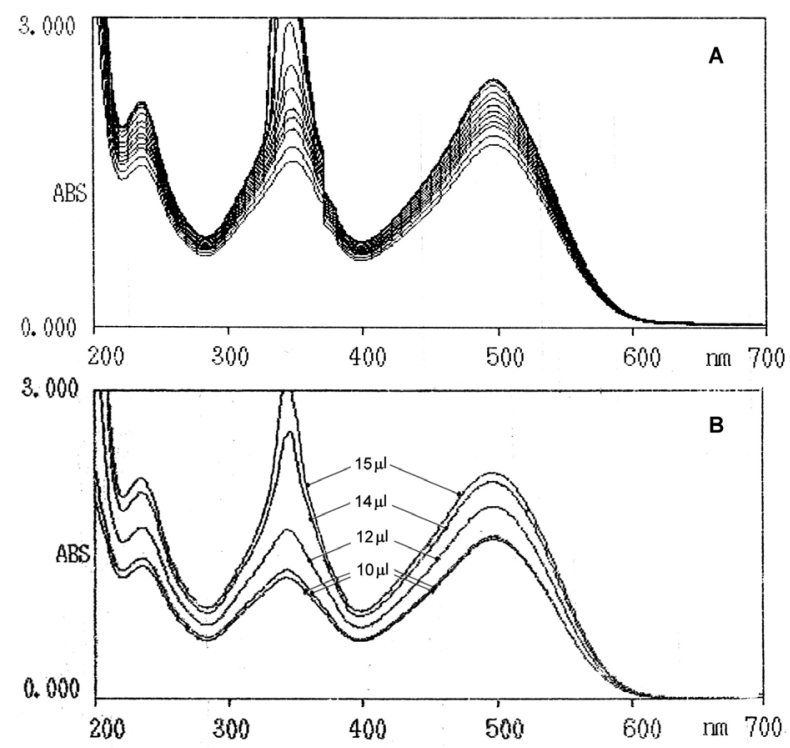

Figure 8. Transition between horizontal and perpendicular electron delocalization in Congo red visualized as non-linear (vs. concentration) change in the spectral absorption.

A - gradual decomposition of supramolecular Congo red structure by step-wise dilution seen as non-linear, selective decrease in UV absorption band, down to the level of the standard spectrum. $\mathrm{B}$ - detailed visualization of shifting from the linear to non-linear Congo red absorption increase upon increasing dye concentration by addition of small but critical for spectral transition portions of stock dye solution $(2.5 \mathrm{mg} / \mathrm{ml})$.

$35 \mathrm{~nm}$, depending on conditions employed. Compare for example spectra in Fig. 9, Fig. 10 and Fig. 11.

Support for spectral visualization of self-assembling has also come from using two isomeric dyes: Evans Blue (EB) and Trypan Blue (TB), which differ in self-assembling tendency. That tendency is high for EB and poor for TB because of a disadvantaged position of sulfonic groups in the TB molecules. Figure 10 shows the different respective spectral changes at increasing concentration of the dyes obtained by addition of a stock dye solution $(3.2 \mathrm{mg} / \mathrm{ml}$ of buffer).

\section{Reinforcement of supramolecular Congo red structures by scaffolding}

Interestingly, relatively stable micelles may also be obtained under lower dye concentrations; however, this re- 


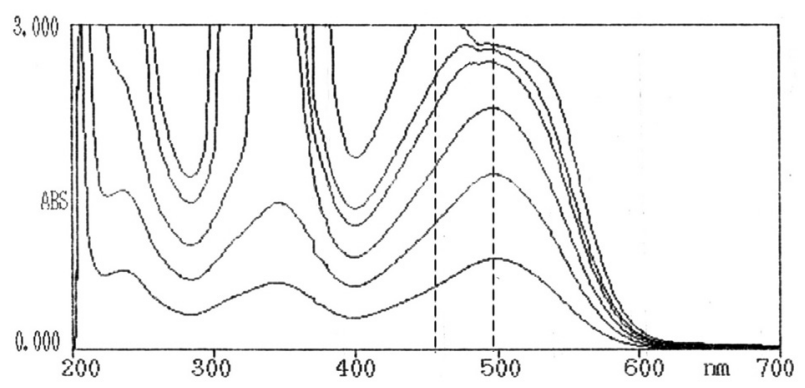

Figure 9. Spectrophotometric evidence of the influence of rapidly increasing UV absorption upon visible light absorption of Congo red. The visible light shift toward UV.

Step-wise increase in Congo red concentration obtained by the addition of stock dye solution $(2.5 \mathrm{mg} / \mathrm{ml})$. The effect becomes readily noticeable at high dye concentrations. The experiment was carried out in acetate buffer at pH 5.85 and glycerol 20\%. Straight vertical lines expose the shift of the absorption band.
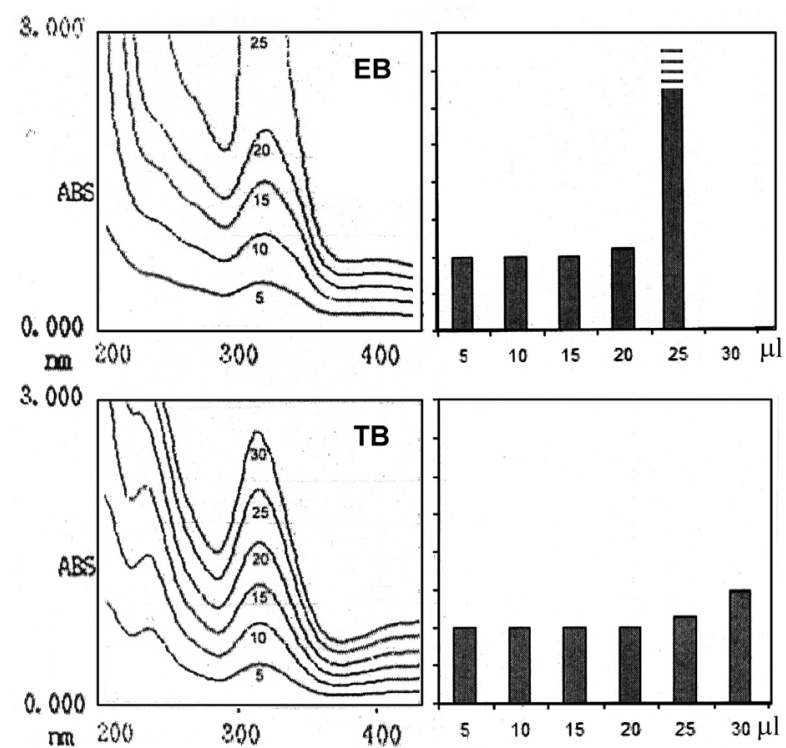

Figure 10. The use of isomeric dyes, Evans blue (EB) and Trypan blue (TB), which differ in self-assembling tendency to reveal spectral effects indicating formation of highly compact supramolecular structure allowing delocalisation of electrons as a reason of the observed non-linear optical changes.

Step-wise increase in concentration was obtained by the addition of equal portions of stock solution of the dye $(3.2 \mathrm{mg} / \mathrm{ml})$. Bars indicate increments in absorption (presented in conventional scale).

quires a suitable structural scaffold - potential candidates include above all the fibrillary structures.

Dextran, a branching alpha-saccharide used in this study, turned out to be inert and largely incapable of binding Congo red. Better results were obtained with gelatin and - most of all - polyvinyl alcohol. The absorbance of Congo red in the UV band increases significantly in the presence of polyvinyl alcohol, reflecting increased association of the dye. Polyvinyl alcohol itself is an associate of fibrillary structures which - at higher concentrations - produce dense, viscous solutions. At lower concentrations, and especially upon heating, the chains dissociate, either partially or entirely. In this state, polyvinyl alcohol may bond to Congo red. This process is reversible and eventually each substance reverts to its original form. If the complex is incubated for a day or longer, it returns to a state of low absorbance. This shows that the mutual cohesion of strands of polyvinyl

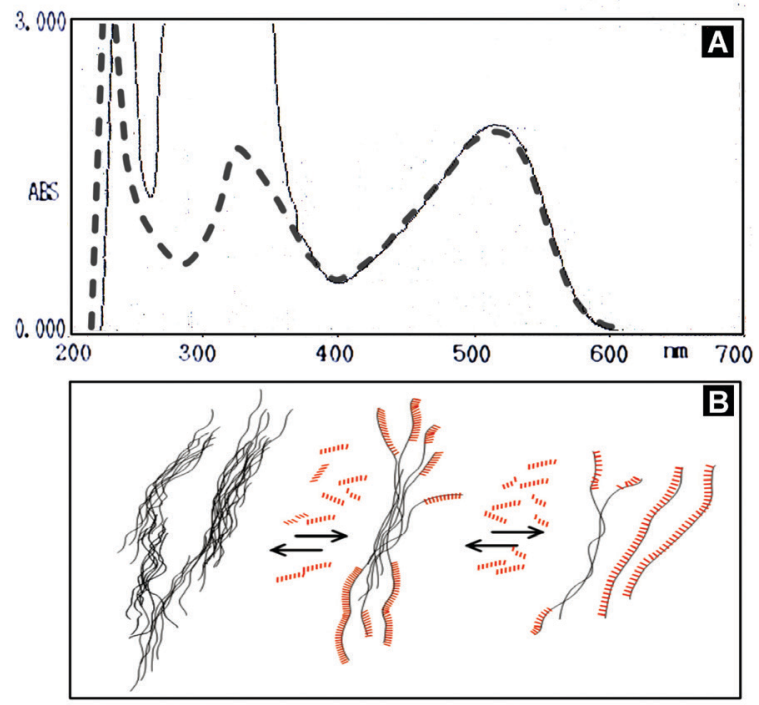

Figure 11. Reversible complexation of Congo red and polyvinyl alcohol.

A - Absorption spectrum of Congo red measured immediately after heating to $100^{\circ} \mathrm{C}$ and than cooling to room temperature - thin line. The same sample 22 hours later - dashed line. B - reversible complexation of Congo red with polyvinyl alcohol - model interpretation. Short red lines - Congo red, black lines - polyvinyl alcohol playing a role of a scaffolding structure.

alcohol overwhelms the stability of the alcohol/dye complex (Fig. 11).

In binding Congo red - which is already a supramolecular system - polyvinyl alcohol effectively lengthens and reinforces the ribbon structure formed by the dye. Covalently stabilized fibrils of polyvinyl alcohol provide a structural scaffold for noncovalently bound aggregates of Congo red. In the absence of alcohol, Congo red forms micellar structures consisting of several molecules. Longer micelles may occasionally appear, but they are rather unstable and break up easily into smaller fragments. Introduction of the scaffold enables much longer associates to emerge.

When Congo red binds to amyloids, the latter fulfill the role of a scaffold (Fig. 12). As it was mentioned before, the dye/amyloid complex has specific spectral

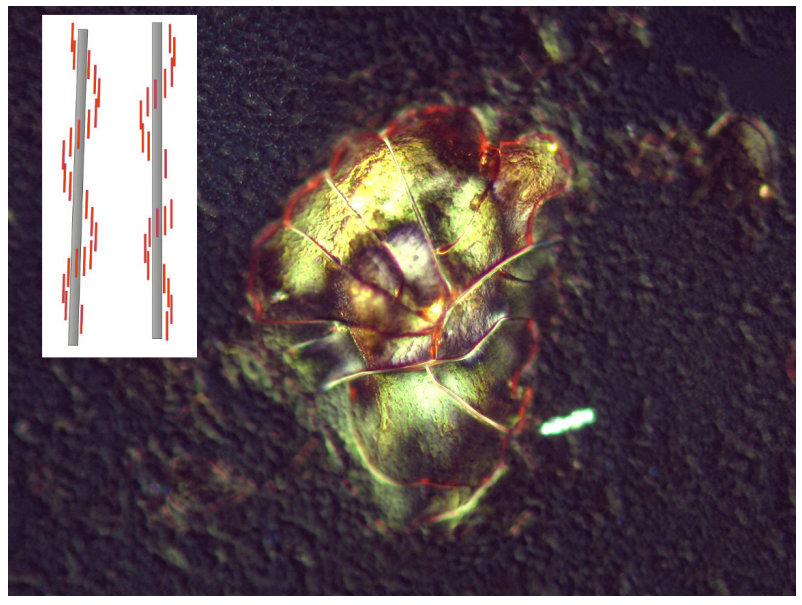

Figure 12. Amyloid fragment stained by Congo red seen under polarised light.

Inset - proposed model of complexation of amyloid with Congo red. Amyloid fibrils providing a structural scaffold for supramolecular Congo red. Short red lines - Congo red molecules, vertical thick lines - amyloid fibrils scaffolding the complex. 


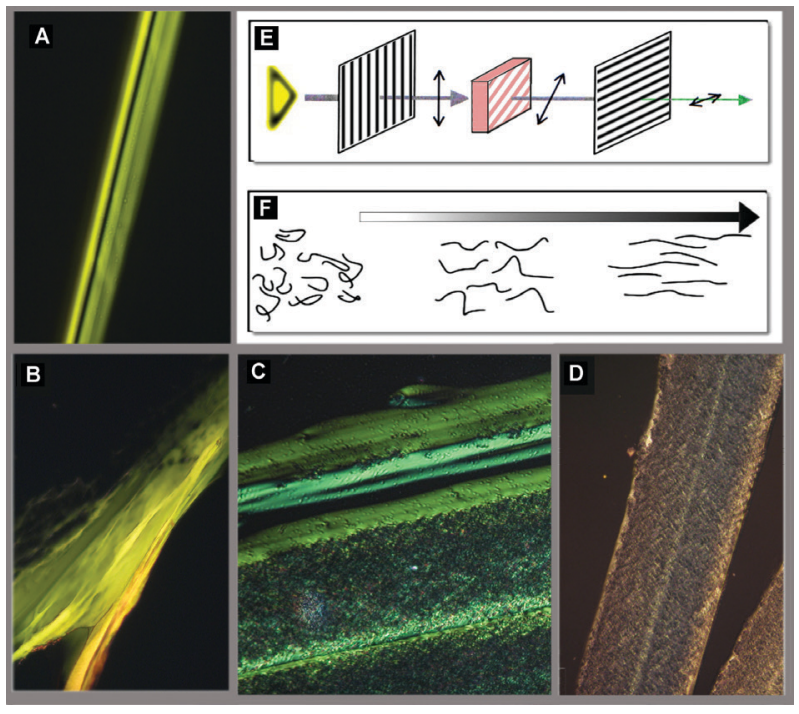

Figure 13. Appearance of optical activity (under polarised light) in a mixture of Congo red and polyvinyl alcohol, dried on a microscopic slide.

Optical activity was elicited by applying pressure with a blunt metal tool and slowly dragging it across a slide. Pictures A, B, C represent colour differentiation from more yellow to more blue. $D$ - optical inertness of Congo red and dextran mixture used instead of polyvinyl alcohol. Inset: E - scheme of optical setup used to measure rotation effects of polarised light. F - mechanism applied to constrain parallel arrangement of scaffold fibers (polyvinyl alcohol - Congo red) by rasping the dried material.

properties which can be studied under polarized light. In particular, spectral analysis of Congo red/amyloid complexes under polarized light reveals an emission in the green region. This emission does not, however, match precisely the absorption spectrum of Congo red in the visual band and usually exhibits a shift towards the yellow region. This is referred to as the apple-green effect (Fig. 12). Causes of this phenomenon remain not fully explainable. Here we present our studies concerning this problem.

\section{The apple-green effect}

It is interesting to speculate whether, and if so - to what extent, the supramolecular nature of Congo red and its capability for electron delocalization contribute to the apple-green effect.

Ordered arrangement of Congo red molecules in complex with amyloids seems to be the main cause of its optical activity. Specific direction in which the light is emitted depends on the direction of fibrils which act as a scaffold (Howie et al., 2008). Such an optically active system may be studied under polarized light. Notably, the color of the emission does not fully correspond to the Congo red absorption spectrum: emissions are often shifted towards longer wavelengths, with the yellow component becoming more pronounced - hence the name "apple-green effect". This spectral shift appears to be caused by several factors, including purely physical ones (Howie et al., 2009). One of them is also the Cotton effect, where the emission of polarized light is preferentially concentrated near the edges of the Congo red absorption spectrum (Benditt et al., 1970). Additionally, a major cause of the observed changes in emission spectrum of Congo red may be attributed to variability in its absorption spectrum. This concerns both, the intensity of absorption and the placement of maxima in the UV and visual bands, which vary significantly depending on

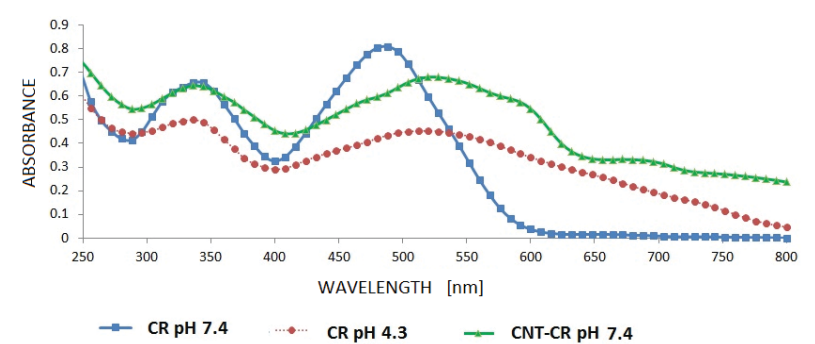

Figure 14. Spectrum of Congo red in complex with carbon nanotubes vs. spectra of Congo red at $\mathrm{pH} 7.4$ and 4.3, respectively. $0.05 \mathrm{M}$ Tris/ $\mathrm{HCl}, 0.9 \% \mathrm{NaCl}$ buffer $\mathrm{pH} 7.4$ and $0.05 \mathrm{M}$ acetate buffer $\mathrm{pH} 4.3$.

conditions, ultimately affecting the emission spectrum of the dye (Fig. 12). This remarkable variability may be explained by massive delocalization of electrons in supramolecular Congo red.

When using matrices which are either capable or incapable of binding Congo red (polyvinyl alcohol, dextran) and inducing complexation of the dye, we may model the spectral shift phenomenon. This is done by exerting mechanical pressure upon a sample placed on a microscopic slide. The sample may be pre-dried, which does not entirely negate its plasticity. Applying gentle pressure with a blunt metal or glass tool which is slowly dragged across the slide forces the scaffold fibers (together with the complexed Congo red) to adopt a parallel arrangement. As a result, the pressure path becomes optically active under polarized light (Hough et al., 2009), which - in the case of polyvinyl alcohol - manifests itself as a green emission but in most cases is shifted towards yellow or blue regions (Fig. 13). When dextran is used as a scaffold, this effect becomes difficult to evoke. It seems that the supramolecular character of Congo red is primarily responsible for its affinity toward amyloids, as well as for the observed spectral effects. At greater concentrations, Congo red begins to exhibit new properties, which are evidenced by increased acidity and spectral phenomena. Both are driven by increased mobility of electrons, with acidification corresponding to areas from which electrons have migrated away.

A reversed effect is observed when Congo red forms complexes with carbon nanotubes. In the course of complexation, nanotubes become soluble and the resulting spectrum resembles one which is characteristic for Congo red in an acidic environment (Fig. 14). Here, nanotubes supply additional electrons to the complexed supramolecular structure of Congo red, counteracting their tendency to dissociate protons from sulfonic groups and instead enabling protons to bind to amino groups (Jagusiak et al., 2018; Sghaier et al., 2016; Ren et al., 2015).

These observations indicate that the supramolecular characteristics of Congo red play a key role in all of the listed interactions.

\section{CONCLUSIONS}

Due to its symmetry and planar structure, Congo red is capable of self-association and forms ribbon-like micelles. At relatively low concentrations these micelles consist of several molecules; however, at higher concentrations longer micelles may form.

Association occurs primarily through interactions of aromatic rings in the central part of the molecule (benzidine derivatives). Face-to-face contact ensures coherence 
of $\pi$-electrons - thus, as the micelle grows, significant delocalization becomes possible, giving rise to a ring current phenomenon. This process polarizes the micelle and alters the $\mathrm{pK}$ of its polar groups. Changes in acidity are not, however, the sole evidence of micellar properties. Further confirmation is provided by UV analysis. In the $300-400 \mathrm{~nm}$ wave length area the absorption remarkably increases, nonlinearly with the increase of the dye concentration, revealing the emergent new properties of elongated ribbons. Variability is also observed in the position of UV and visual absorption bands on the wavelength scale.

This effect is likely due to changeable mutual orientation of the self-assembling Congo red molecules and subsequent rings' contact. Changes in the absorption spectrum have a trickle-down effect on the emission spectrum of Congo red in complex with amyloids.

The presented effects are up to now explained on the physical grounds, in particular by anomalous dispersion of the refractive index (Howie et al., 2009). It seems, however, that chemical factors also play an important role - notably in the context of spectral changes produced by possibly strong delocalization of electrons in the supramolecular Congo red organization.

\section{Acknowledgements}

The authors wish to express many thanks to Piotr Nowakowski for translation and to Romuald Bolesławski for art work.

\section{REFERENCES}

Benditt EP, Eriksen N, Berglund C (1970) Congo red dichroism with dispersed amyloid fibrils, an extrinsic cotton effect. Proc Natl Acad Sci U S A 66: 1044-1051. PMCID: PMC335784

Carter DB, Chou KC (1998) A model for structure-dependent binding of Congo red to Alzheimer beta-amyloid fibrils. Neurobiol Aging 19: 37-40. PMID: 9562501

Chen Y, Tang C, Zhang J, Gong M, Su B, Qiu F (2015) Self-assembling surfactant-like peptide $\mathrm{A} 6 \mathrm{~K}$ as potential delivery system for hydrophobic drugs. Int J Nanomedicine 10: 847-858. https://doi. org/10.2147/IJN.S71696

Chiti F, Dobson CM (2017) Protein Misfolding, Amyloid Formation, and Human Disease: A Summary of Progress Over the Last Decade. Annu Rev Biochem 86: 27-68. https://doi.org/10.1146/annurevbiochem-061516-045115.

Colon W, Kelly JW (1992) Partial denaturation of transthyretin is sufficient for amyloid fibril formation in vitro. Biochemistry 31: 8654-8660. PMID: 1390650

Dygut J, Kalinowska B, Banach M, Piwowar M, Konieczny L, Roterman I (2016) Structural interface forms and their involvement in stabilization of multidomain proteins or protein complexes. Int $J$ Mol Sci 17. pii: E1741

Frid P, Anisimov SV, Popovic N (2007) Congo red and protein aggregation in neurodegenerative diseases. Brain Res Rev 53: 135-160. https://doi.org/10.1016/j.brainresrev.2006.08.001

Harper JD, Wong SS, Lieber CM, Lansbury PT (1997) Observation of metastable Abeta amyloid protofibrils by atomic force microscopy. Chem Biol 4: 119-125. PMID: 9190286

Herbst S, Soberats B, Leowanawat P, Stolte M, Lehmann M, Würthner F (2018) Self-assembly of multi-stranded perylene dye J-aggregates in columnar liquid-crystalline phases. Nat Commun 9: 2646. https:// doi.org/10.1038/s41467-018-05018-6

Hough LE, Spannuth M, Nakata M, Coleman DA, Jones CD, Dantlgraber $G$, Tschierske C, Watanabe J, Körblova E, Walba DM, Maclennan JE, Glaser MA, Clark NA (2009) Chiral isotropic liquids from achiral molecules. Science 325: 452-456. https://doi. org/10.1126/science.1170028

Howie AJ, Brewer DB, Howell D, Jones AP (2008) Physical basis of colors seen in Congo red-stained amyloid in polarized light. Lab Invest 88: 232-242. https://doi.org/10.1038/labinvest.3700714

Howie AJ, Brewer DB (2009) Optical properties of amyloid stained by Congo red: history and mechanisms. Micron 40: 285-301. https:// doi.org/10.1016/i.micron.2008.10.002

Huang K, Maiti NC, Phillips NB, Carey PR, Weiss MA (2006) Structure-specific effects of protein topology on cross-beta assembly: studies of insulin fibrillation. Biochemistry 45: 10278-10293. https:// doi.org/10.1021/bi060879g

Jagusiak A, Rybarska J, Piekarska B, Stopa B, Konieczny L (2018) Supramolecular Congo Red as specific ligand of antibodies engaged in immune complex. In Self-Assembled Molecules - New Kind of Protein Ligands. Roterman I, Konieczny L eds, pp. 21-41 Springer. ISBN 978-3-319-65639-7

Jagusiak A, Piekarska B, Pańczyk T, Jemioła-Rzemińska M, Bielańska E, Stopa B, Zemanek G, Rybarska J, Roterman I, Konieczny L. (2017) Dispersion of single-wall carbon nanotubes with supramolecular Congo red - properties of the complexes and mechanism of the interaction. Beilstein J Nanotechnol 8: 636-648. https://doi. org/10.3762/bjnano.8.68. eCollection 2017

Kalinowska B, Banach M, Wiśniowski Z, Konieczny L, Roterman I. (2017) Is the hydrophobic core a universal structural element in proteins? J Mol Model 23: 205. https://doi.org/10.1007/s00894-0173367-z

Kauzmann W (1959) Some factors in the interpretation of protein denaturation. Adv Protein Chem 14: 1-63. PMID: 14404936

Kayed R, Head E, Thompson JL, McIntire TM, Milton SC, Cotman CW, Glabe CG (2003) Common structure of soluble amyloid oligomers implies common mechanism of pathogenesis. Science $\mathbf{3 0 0}$ : 486-489. https://doi.org/10.1126/science.1079469

Khurana R, Gillespie JR, Talapatra A, Minert LJ, Ionescu-Zanetti C, Millett I, Fink AL (2001) Partially folded intermediates as critical precursors of light chain amyloid fibrils and amorphous aggregates. Biochemistry 40: 3525-3535. PMID: 11297418

Klunk WE, Pettegrew JW, Abraham DJ (1989) Quantitative evaluation of congo red binding to amyloid-like proteins with a beta-pleated sheet conformation. I Histochem Cytochem 37: 1273-1281. PMID: 2666510

Krishnan R, Goodman JL, Mukhopadhyay S, Pacheco CD, Lemke EA, Deniz AA, Lindquist S (2012) Conserved features of intermediates in amyloid assembly determine their benign or toxic states. Proc Natl Acad Sci U S A 109: 11172-11177. https://doi.org/10.1073/ pnas.1209527109

Lendel C, Bolognesi B, Wahlström A, Dobson CM, Gräslund A (2010) Detergent-like interaction of Congo red with the amyloid beta peptide. Biochemistry 49: 1358-1360. https://doi.org/10.1021/bi902005t

Linke RP, Tischendorf FW, Zucker-Franklin D, Franklin EC (1973) The formation of amyloid-like fibrils in vitro from Bence Jones Proteins of the VlambdaI subclass. J Immunol 111: 24-26. PMID: 4123371

Liu D, Wüthrich K (2016) Ring current shifts in (19)F-NMR of membrane proteins. J Biomol NMR 65: 1-5. https://doi.org/10.1007/ s10858-016-0022-4

Ma X, Liu L, Mao X, Niu L, Deng K, Wu W, Li Y, Yang Y, Wang C (2009) Amyloid beta (1-42) folding multiplicity and single-molecule binding behavior studied with STM. J Mol Biol 388: 894-901. https://doi.org/10.1016/j.jmb.2009.03.054

Modler AJ, Gast K, Lutsch G, Damaschun G (2003) Assembly of amyloid protofibrils via critical oligomers - a novel pathway of amyloid formation. J Mol Biol 325: 135-148. PMID:12473457

Nelson R, Eisenberg D (2006) Recent atomic models of amyloid fibril structure. Curr Opin Struct Biol 16: 260-265. PMID: 16563741

Nummelin S, Selin M, Legrand S, Ropponen J, Seitsonen J, Nykänen A, Koivisto J, Hirvonen J, Kostiainen MA, Bimbo LM (2017) Modular synthesis of self-assembling Janus-dendrimers and facile preparation of drug-loaded dendrimersomes. Nanoscale 9: 7189-7198. https://doi.org/10.1039/c6nr08102a

Pratim Bose P, Chatterjee U, Xie L, Johansson J, Göthelid E, Arvidsson PI (2010) Effects of Congo red on a $\beta(1-40)$ fibril formation process and morphology. ACS Chem Neurosic 1: 315-324. https:// doi.org/10.1021/cn900041x

Ren P, Zheng A, Xiao J, Pan X, Bao X (2015) Exploring the ring current of carbon nanotubes by first-principles calculations. Chem Sci $\mathbf{6}$ : 902-908. https://doi.org/10.1039/c4sc01996b

Roterman I, Konieczny L (2018) Self-Assembled Molecules - New Kind of Protein Ligands. Springer Open, ISBN 978-3-319-65639-7

Sahakyan AB, Vendruscolo M (2013) Analysis of the contributions of ring current and electric field effects to the chemical shifts of RNA bases. J Phys Chem B 117: 1989-1998. https://doi.org/10.1021/ jp3057306

Schormann N, Murrell JR, Benson MD (1998) Tertiary structures of amyloidogenic and non-amyloidogenic transthyretin variants: new model for amyloid fibril formation. Amyloid 5: 175-187. PMID: 9818054

Serpell LC (2000) Alzheimer's amyloid fibrils: structure and assembly. Biochim Biophys Acta 1502: 16-30. PMID: 10899428

Sghaier T, Le Liepvre S, Fiorini C, Douillard L, Charra F (2016) Optical absorption signature of a self-assembled dye monolayer on graphene. Beilstein J Nanotechnol 7: 862-868. https://doi.org/10.3762/ bjnano.7.78. eCollection 2016

Skowronek M, Konieczny L, Rybarska J, Piekarska B, Szneler E, Bakalarski G, Roterman I (1998) Self-assembly of Congo red - a theoretical and experimental approach to identify its supramolecu- 
lar organisation in water and salt solutions. Biopolymers 46: 267-281. https://doi.org/10.1002/(SICI)1097-0282(19981015)46:5<267::AIDBIP1>3.0.CO;2-N

Spólnik P, Król M, Stopa B, Konieczny L, Piekarska B, Rybarska J, Zemanek G, Jagusiak A, Piwowar P, Szoniec G, Roterman I (2011) Influence of the electric field on supramolecular structure and properties of amyloid-specific reagent Congo red. Eur Biophys J 40: 1187-1196. https://doi.org/10.1007/s00249-011-0750-z

Stopa B, Rybarska J, Drozd A, Konieczny L, Król M, Lisowski M, Piekarska B, Roterman I, Spólnik P, Zemanek G (2006) Albumin binds self-assembling dyes as specific polymolecular ligands. Int J Biol Macromol 40: 1-8. https://doi.org/10.1016/j.ijbiomac.2006.05.002

Stopa B, Piekarska B, Konieczny L, Król M, Rybarska J, Jagusiak A, Spólnik P, Roterman I, Urbanowicz B, Piwowar P, Lewiński K
(2010) Formation of amyloid-like aggregates through the attachment of protein molecules to a Congo red scaffolding framework ordered under the influence o fan electric field. Central Europe J Chem 8: 4150. https://doi.org/10.2478/s11532-009-0107-y

Turnell WG, Finch JT (1992) Binding of the dye congo red to the amyloid protein pig insulin reveals a novel homology amongst amyloid-forming peptide sequences. J Mol Biol 227: 1205-1223. PMID: 1433294

Wu C, Scott J, Shea JE (2012) Binding of Congo red to amyloid protofibrils of the Alzheimer $A \beta(9-40)$ peptide probed by molecular dynamics simulations. Biophys J 103: 550-557. https://doi. org/10.1016/j.bpj.2012.07.008 